\title{
Norm-Based Joint Transmit/Receive Antenna Selection Aided and Two-Tier Channel Estimation Assisted STSK Systems
}

\author{
Peichang Zhang ${ }^{1}$, Sheng Chen ${ }^{1,2}$, Chen Dong ${ }^{1}, \mathrm{Li} \mathrm{Li}^{1}$, and Lajos Hanzo ${ }^{1}$ \\ ${ }^{1}$ Electronics and Computer Science, University of Southampton, Southampton SO17 1BJ, U.K. \\ E-mails: $\{$ pz3g09, sqc, cd2g09, 115e08, lh\}@ecs.soton.ac.uk \\ ${ }^{2}$ Faculty of Engineering, King Abdulaziz University, Jeddah 21589, Saudi Arabia
}

\begin{abstract}
We propose a simple yet effective norm-based joint transmit and receive antenna selection (NBJTRAS) assisted and two-tier channel estimation (TTCE) aided space-time shift keying (STSK) system, which is capable of significantly outperforming the conventional STSK system, while efficiently utilising available radio frequency (RF) chains. Specifically, the NBJTRAS carries out antenna selection based on the channel estimation (CE) generated using a low-complexity training based least square channel estimator by reusing RF chains. The selected sub-channel matrix is further refined by an efficient semi-blind $\mathrm{CE}$ and data detection scheme. Our simulation results show that only a few iterations are sufficient for the TTCE scheme to approach the optimal maximum-likelihood detection performance associated with perfectly channel state information.

Index Terms-Multi-input multi-output, joint transmit/receive
\end{abstract} antenna selection, space-time shift keying, channel estimation

\section{INTRODUCTION}

Although multiple-input multiple-output (MIMO) systems are capable of improving system's reliability and capacity, they require large number of radio frequency (RF) chains, which leads to high power consumption and hardware costs as well as high complexity in channel estimation (CE). Antenna selection (AS) offers a low-cost alternative to reduce the number of RF chains utilised at transmitter and/or receiver, while retaining the significant advantages of MIMO systems. Generally, AS may be classified into three categories, namely, transmit AS (TxAS), receive AS (RxAS) and joint transmit and receive AS (JTRAS) [1]. The TxAS schemes for MIMO systems were studied in [2]-[4]. More explicitly, two TxAS techniques were proposed and compared in [2] for spatial modulation (SM) systems, where it was shown that the capacity optimised AS scheme outperformed the Euclidean distance optimised AS one. Three AS criteria were proposed for space-shift keying (SSK) systems in [3], which were the max-norm based AS (ASC1), the maximum norm difference based AS (ASC2), and the hybrid scheme combining ASC1 and ASC2. The simulation results of [3] showed that AS techniques were capable of improving the performance of SSK aided MIMO systems, and ASC1 outperformed both ASC2 and the hybrid design. The RxAS schemes for MIMO systems were studied in [5]-[8]. More specifically, the work [5] proposed an optimal RxAS scheme for space-time trellis codes, which selected receive antennas with the highest instantaneous signal-to-noise ratio (SNR). A RxAS method was proposed for V-BLAST systems

The financial supports under the EU Concerto project and the European Research Council's Advanced Fellow Grant are gratefully acknowledged. in [6], where it was shown that the system performance was improved with the aid of AS in terms of block error rate.

As a hybrid version of TxAS and RxAS, JTRAS schemes were investigated in [9]-[17], where they were observed to be capable of improving the system performance while maintaining low transceiver hardware complexity. Moreover, it is well-known that the optimal capacity-based AS usually requires exhaustive search over all the possible subsets of the full channel matrix, which becomes impractical for the system with a large number of transmit and/or receive antennas [17]. Some sub-optimal capacity-based AS techniques were proposed in [13], [14], [17], which were capable of reducing the AS complexity at the cost of certain performance loss. As another efficient yet simple category of AS algorithms, norm-based AS (NBAS) techniques, were investigated in [12], [15], [16], where it was shown that NBAS algorithms were capable of approaching the performance of capacity based AS techniques, while imposing lower system complexity.

Most existing AS techniques [2]-[17] assume that the channel state information (CSI) is perfectly known at transmitter and/or receiver. However, the acquisition of accurate MIMO CSI imposes an excessive pilot-overhead, which not only significantly erodes the system's achievable throughput but also results in an excessive CE complexity. The training based minimum mean square error (MMSE) channel estimator was employed in [18] for RxAS aided space-time coded MIMO systems, which only considered selecting a single receive antenna. In [19], the training based linear MMSE channel estimator was investigated for MIMO-OFDM systems with RxAS, where AS was only performed based on the received signal power occurring prior to channel estimation. As a unified MIMO architecture that includes SM [20] and SSK [21] as its special cases, the space-time shift-keying (STSK) was conceived in [22], and a low-complexity semi-blind scheme for STSK systems [23] is capable of accurately estimating the CSI without imposing high training overhead.

Against the above background, our novel contribution is twofold. Firstly, we propose a new norm-based JTRAS (NBJTRAS) aided STSK system, which significantly outperforms the conventional STSK system in terms of bit error rate (BER), given the CSI, while maintaining a low system complexity. In particular, we define the AS factor, which indicates the additional diversity order attained by the NBJTRAS aided STSK system. Secondly, we propose a novel two-tier CE (TTCE) scheme for assisting the NBJTRAS based STSK system. 
Specifically, in tier one, a low-complexity training based least square CE (LSCE) with RF chain reuse is performed to obtain a rough $\mathrm{CE}$ of the full channel set by only utilizing a small number of training symbol blocks, for the sake of maintaining a high system throughput. The overhead of feedforward and feedback in tier one is minimal as they only involve antenna indexes. The NBJTRAS is carried out based on this initial CE. In tier two, a semi-blind joint $\mathrm{CE}$ and data detection scheme [24] is used to further refine the CE of the selected channel subset. The low-complexity single-stream maximum likelihood (ML) data detection for the STSK system is carried out based on the selected channel subset found in the tier-one stage, and the detected data are re-modulated and used for further decision-directed CE (DDCE). Our simulation results show that with the aid of this proposed TTCE, the system's performance converges in a few iterations to the optimal ML performance associated with perfect CSI.

\section{SySTEM OVERVIEW}

Boldface capital and lower-case letters stand for matrices and column vectors, respectively. The inverse operation is given by ()$^{-1}$, while the transpose and conjugate transpose operators are given by ()$^{\mathrm{T}}$ and ()$^{\mathrm{H}}$, respectively. The norm and magnitude operators are denoted by \|\| and | |, respectively. The $M \times M$ identity matrix is denoted by $\boldsymbol{I}_{M}$, and $\boldsymbol{H}(i, j)$ is the $i$ th-row and $j$ th-column element of $\boldsymbol{H}$.

\section{A. STSK System Model}

We consider a frequency-flat Rayleigh fading environment. Let $\operatorname{STSK}\left(N_{T}, N_{R}, T_{n}, Q, L\right)$ be the STSK system employing $L$-phase shift keying (PSK) or $L$-quadrature amplitude modulation (QAM), where $N_{T}$ and $N_{R}$ are the numbers of transmit and receive antennas, respectively, while $T_{n}$ is the number of time slots occupied by the STSK signal block and $Q$ is the number of dispersion matrices employed. The numbers of transmit and receive RF chains are given by $L_{T}$ and $L_{R}$, respectively. Let $i$ denote the STSK block index. At the STSK transmitter, the information bit sequence is firstly converted to a number of blocks with the number of bits per block given by $N=\log _{2}(Q)+\log _{2}(\mathcal{L})$. The first $\log _{2}(Q)$ bits of the $i$ th block are used to choose the dispersion matrix $\boldsymbol{A}(i)$ from the $Q$ preassigned dispersion matrices $\left\{\boldsymbol{A}_{q} \in \mathbb{C}^{N_{T} \times T_{n}}, 1 \leq q \leq Q\right\}$, while the remaining $\log _{2}(L)$ bits are mapped to the complexvalued symbol $s(i) \in\left\{s_{l}, 1 \leq l \leq L\right\}$ of the $L$-PSK/QAM [22]. In this way, a total of $N$ source bits are mapped to a single STSK signal block $\boldsymbol{S}(i) \in \mathbb{C}^{N_{T} \times T_{n}}$ with

$$
\boldsymbol{S}(i)=s(i) \boldsymbol{A}(i) \text {. }
$$

The $m$ th row of $\boldsymbol{S}(i)$ is transmitted through the $m$ th transmit antenna in the $T_{n}$ time slots.

The corresponding received signal block $\boldsymbol{Y}(i) \in \mathbb{C}^{N_{R} \times T_{n}}$ can be expressed as [22]

$$
\boldsymbol{Y}(i)=\boldsymbol{H} \boldsymbol{S}(i)+\boldsymbol{V}(i),
$$

where $\boldsymbol{H} \in \mathbb{C}^{N_{R} \times N_{T}}$ is the MIMO channel matrix, whose elements obey the complex-valued Gaussian distribution of zero-mean and unit variance, denoted as $\mathcal{C N}(0,1)$, while each element of the additive white Gaussian noise (AWGN) matrix $\boldsymbol{V}(i) \in \mathbb{C}^{N_{R} \times T_{n}}$ obeys the complex-valued Gaussian distribution of $\mathcal{C N}\left(0, N_{\mathrm{o}}\right)$ with $N_{\mathrm{o}}$ being the AWGN power. Define the equivalent transmitted signal vector $\boldsymbol{k}(i)$ as

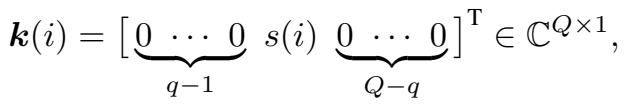

where $q$ indicates the corresponding dispersion matrix that is activated for the $i$ th STSK block. The total number of legitimate transmit signal vectors for $\boldsymbol{k}(i)$ is $L \cdot Q$, and we have $\boldsymbol{k}(i) \in\left\{\boldsymbol{k}_{q, l}, 1 \leq q \leq Q, 1 \leq l \leq L\right\}$ with

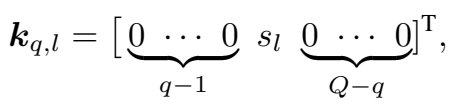

where $s_{l}$ is the $l$ th symbol in the $L$-PSK/QAM. By defining

$$
\begin{aligned}
\overline{\boldsymbol{y}}(i) & =\operatorname{vec}(\boldsymbol{Y}(i)) \in \mathbb{C}^{N_{R} T_{n} \times 1}, \\
\overline{\boldsymbol{H}} & =\boldsymbol{I}_{T_{n}} \otimes \boldsymbol{H} \in \mathbb{C}^{N_{R} T_{n} \times N_{T} T_{n}}, \\
\boldsymbol{\Upsilon} & =\left[\operatorname{vec}\left(\boldsymbol{A}_{1}\right) \operatorname{vec}\left(\boldsymbol{A}_{2}\right) \cdots \operatorname{vec}\left(\boldsymbol{A}_{Q}\right)\right] \in \mathbb{C}^{N_{T} T_{n} \times Q}, \\
\overline{\boldsymbol{v}}(i) & =\operatorname{vec}(\boldsymbol{V}(i)) \in \mathbb{C}^{N_{R} T_{n} \times 1},
\end{aligned}
$$

the equivalent system model is given as [22]

$$
\overline{\boldsymbol{y}}(i)=\overline{\boldsymbol{H}} \boldsymbol{r} \boldsymbol{k}(i)+\overline{\boldsymbol{v}}(i) .
$$

Given $\boldsymbol{H}$, a low-complexity single-stream based ML detector can be applied, since the equivalent system model (9) is free from interchannel interference [22]. Let $(q, l)$ be the input index of the dispersion matrix and modulated symbol that have been selected at the transmitter for the $i$ th STSK signal block, the ML estimate of $(q, l)$ is given by

$$
(\hat{q}, \hat{l})=\arg \min _{1 \leq \tilde{q} \leq Q, 1 \leq \tilde{l} \leq L}\left\|\overline{\boldsymbol{y}}(i)-\overline{\boldsymbol{H}} \boldsymbol{\Upsilon} \boldsymbol{k}_{\tilde{q}, \tilde{l}}\right\|^{2} .
$$

\section{B. NBJTRAS Aided STSK System}

The proposed NBJTRAS aided STSK system is depicted in Fig. 1, where for the time being we assume that the full channel matrix $\boldsymbol{H}$ is known. Since the numbers of the RF chains at the transmitter and receiver are $L_{T}<N_{T}$ and $L_{R}<N_{R}$, respectively, the resulting STSK system has the configuration $\operatorname{STSK}\left(L_{T}, L_{R}, T_{n}, Q, L\right)$ with the communication occurring on the selected subset channel matrix $\boldsymbol{H}_{\text {sub }} \in \mathbb{C}^{L_{R} \times L_{T}}$.

Generally speaking, larger channel gain yields better system performance. This leads to the NBAS approach which selects the transmit and receive antennas related to the subset channel matrix with the highest channel norm. Let $\widetilde{\boldsymbol{H}}_{\text {sub }} \in \mathbb{C}^{L_{R} \times L_{T}}$ be the subset candidates of the full channel matrix $\boldsymbol{H}$. The selected subset $\boldsymbol{H}_{\text {sub }}$ based on the NBAS criterion is found by solving the optimization

$$
\boldsymbol{H}_{s u b}=\arg \max _{\widetilde{\boldsymbol{H}}_{s u b} \subset \boldsymbol{H}} \sum_{n_{t}=1}^{L_{T}} \sum_{n_{r}=1}^{L_{R}}\left\|\widetilde{\boldsymbol{H}}_{s u b}\left(n_{r}, n_{t}\right)\right\|^{2} .
$$

Solving the above optimization by exhaustive search requires to evaluate the norms of the $\mathrm{C}_{N_{R}}^{L_{R}} \times \mathrm{C}_{N_{T}}^{L_{T}}$ candidate subset matrices, where $\mathrm{C}_{k}^{n}=\frac{k !}{n !(k-n) !}, \mathrm{C}_{N_{R}}^{L_{R}}$ and $\mathrm{C}_{N_{T}}^{L_{T}}$ are the 


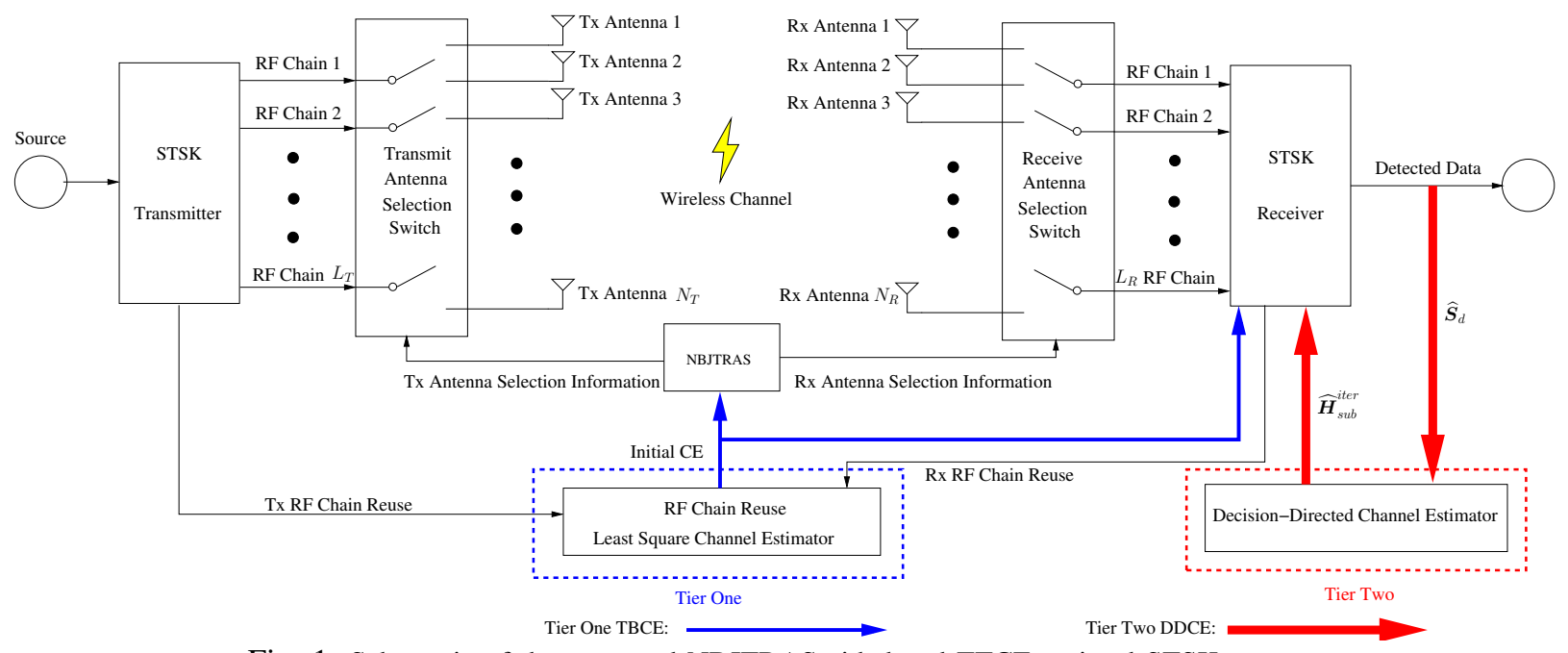

Fig. 1: Schematic of the proposed NBJTRAS aided and TTCE assisted STSK systems.

row-dimension and column-dimension combinations of $\boldsymbol{H}_{s u b}$, respectively. We now present a novel NBJTRAS scheme to solve the optimization (11) at a much lower complexity.

Given the full channel matrix $\boldsymbol{H} \in \mathbb{C}^{N_{R} \times N_{T}}$, without loss of generality, assume $\mathrm{C}_{N_{R}}^{L_{R}}<\mathrm{C}_{N_{T}}^{L_{T}}$. Our NBJTRAS algorithm accomplishes the optimization in the following two steps.

Step 1): Row Dimension Operations.

Let $i_{r} \in\left\{1,2, \cdots, \mathrm{C}_{N_{R}}^{L_{R}}\right\}$ be the row combination index, and the row indices corresponding to the $i_{r}$ th sub-matrix $\boldsymbol{H}_{i_{r}} \in \mathbb{C}^{L_{R} \times N_{T}}$ be given by $\boldsymbol{l}_{i_{r}}=\left[l_{i_{r}}^{1} l_{i_{r}}^{2} \cdots l_{i_{r}}^{L_{R}}\right]^{\mathrm{T}}$. Then

$$
\boldsymbol{H}_{i_{r}}=\left[\begin{array}{c}
\boldsymbol{h}_{l_{i_{r}}^{\mathrm{T}}}^{\mathrm{T}} \\
\boldsymbol{h}_{l_{i_{r}}^{\mathrm{T}}}^{\mathrm{T}} \\
\vdots \\
\boldsymbol{h}_{l_{i_{r}}^{L_{2}}}^{\mathrm{T}}
\end{array}\right]=\left[\begin{array}{ccc}
\boldsymbol{H}_{i_{r}}(1,1) & \cdots & \boldsymbol{H}_{i_{r}}\left(1, N_{T}\right) \\
\boldsymbol{H}_{i_{r}}(2,1) & \cdots & \boldsymbol{H}_{i_{r}}\left(2, N_{T}\right) \\
\vdots & \cdots & \vdots \\
\boldsymbol{H}_{i_{r}}\left(L_{R}, 1\right) & \cdots & \boldsymbol{H}_{i_{r}}\left(L_{R}, N_{T}\right)
\end{array}\right]
$$

where $\boldsymbol{h}_{x}^{\mathrm{T}}$ is the $x$ th row of $\boldsymbol{H}$. Computing

$$
m_{i_{r}}^{x}=\sum_{j=1}^{L_{R}}\left|\boldsymbol{H}_{i_{r}}(j, x)\right|^{2}, 1 \leq x \leq N_{T},
$$

where $m_{i_{r}}^{x}$ represents the magnitude of the $x$ th column in $\boldsymbol{H}_{i_{r}}$, yields the norm metric vector

$$
\boldsymbol{m}_{i_{r}}^{\mathrm{T}}=\left[m_{i_{r}}^{1} m_{i_{r}}^{2} \cdots m_{i_{r}}^{N_{T}}\right] .
$$

Applying (14) to all the $\mathrm{C}_{N_{R}}^{L_{R}}$ possible combinations leads to the norm metric matrix $M \in \mathbb{C}^{\mathrm{C}_{N_{R}}^{L_{R}} \times N_{T}}$ given by

$$
\boldsymbol{M}=\left[\begin{array}{c}
\boldsymbol{m}_{1}^{\mathrm{T}} \\
\boldsymbol{m}_{2}^{\mathrm{T}} \\
\vdots \\
\boldsymbol{m}_{\mathrm{C}_{N_{R}}^{L_{R}}}^{\mathrm{T}}
\end{array}\right]=\left[\begin{array}{cccc}
m_{1}^{1} & m_{1}^{2} & \cdots & m_{1}^{N_{T}} \\
m_{2}^{1} & m_{2}^{2} & \cdots & m_{2}^{N_{T}} \\
\vdots & \vdots & \cdots & \vdots \\
m_{\mathrm{C}_{N_{R}}^{L_{R}}}^{1} & m_{\mathrm{C}_{N_{R}}^{L_{R}}}^{2} & \cdots & m_{\mathrm{C}_{N_{R}}^{N_{T}}}^{L_{R}}
\end{array}\right] .
$$

Step 2): Column Dimension Operations.

Find the largest $L_{T}$ elements in the $i_{r}$ th row of $M$ and sum them up, which is denoted as $m_{\max }^{i_{r}}$, as well as record the column indices of these $L_{T}$ elements in the index vector $\boldsymbol{l}_{i_{c}}\left(i_{r}\right)=\left[l_{i_{c}}^{1}\left(i_{r}\right) l_{i_{c}}^{2}\left(i_{r}\right) \cdots l_{i_{c}}^{L_{T}}\left(i_{r}\right)\right]^{\mathrm{T}}$. This produces the max-norm metric vector

$$
\boldsymbol{m}_{\max }^{\mathrm{T}}=\left[m_{\max }^{1} m_{\max }^{2} \cdots m_{\max }^{\mathrm{C}_{N_{R}}^{L_{R}}}\right] .
$$

Next find

$$
\bar{i}_{r}=\arg \max _{1 \leq i_{r} \leq \mathrm{C}_{N_{R}}^{L_{R}}} m_{\max }^{i_{r}}
$$

Then selected transmit and receive antenna indices are specified by $\boldsymbol{l}_{i_{c}}\left(\bar{i}_{r}\right)$ and $\boldsymbol{l}_{\bar{i}_{r}}$, respectively, and the corresponding subset channel matrix $\boldsymbol{H}_{\text {sub }}$ is the optimal solution of (11).

The complexity of the NBJTRAS is $C_{\text {NBJTRAS }} \approx$ $\mathcal{O}\left(\left(N_{T} \cdot L_{R}\right) \cdot \mathrm{C}_{N_{R}}^{L_{R}}\right)$, which is much smaller than $C_{\mathrm{ES}} \approx$ $\mathcal{O}\left(\left(L_{R} \cdot L_{T}\right) \cdot\left(\mathrm{C}_{N_{T}}^{L_{T}} \cdot \mathrm{C}_{N_{R}}^{L_{R}}\right)\right)$ of the exhaustive search. If $\mathrm{C}_{N_{R}}^{L_{R}}>\mathrm{C}_{N_{T}}^{L_{T}}$, the NBJTRAS starts with Step 1) of Column Dimension Operations followed by Step 2) of Row Dimension Operations, and the complexity of this algorithm is $\mathcal{O}\left(\left(N_{R} \cdot L_{T}\right) \cdot \mathrm{C}_{N_{T}}^{L_{T}}\right)$.

Given $L_{R}$ and $L_{T}$, the achievable multiplexing gain of the STSK system is fixed. We now define the AS factor as

$$
f_{A S}\left(N_{T}, N_{R}\right)=\frac{N_{T}+N_{R}}{L_{T}+L_{R}},
$$

which is the diversity order attained, compared with the conventional $\operatorname{STSK}\left(L_{T}, L_{R}, T_{n}, Q, L\right)$ without AS.

\section{Two-TIER CHANNEL ESTIMATION FOR NBJTRAS}

The TTCE scheme consists of Tier-One training based CE (TBCE) and Tier-Two DDCE as illustrated in Fig. 1.

\section{A. Tier One: TBCE}

We adopt the training based LSCE with a very small number of training blocks in tier one to maintain a high throughput at the cost of a poor CE. According to the study [25], AS is relatively insensitive to $\mathrm{CE}$ error and, therefore, this inaccurate $\mathrm{CE}$ is adequate for the NBJTRAS scheme to carry out the AS task. Because the numbers of the RF chains available at transmitter/receiver are smaller than those of transmit/receive 
antennas, RF chains are reused in the estimation of the full channel matrix $\boldsymbol{H} \in \mathbb{C}^{N_{R} \times N_{T}}$. For simplicity and without loss of generality, we assume that both the ratios of $\frac{N_{T}}{L_{T}}$ and $\frac{N_{R}}{L_{R}}$ are integers. Then the number of the subset channel matrices that need to be estimated is $\frac{N_{T}}{L_{T}} \times \frac{N_{R}}{L_{R}}$. Specifically, we need to estimate the subset channel matrices $\boldsymbol{H}_{\text {est }}^{(i, j)} \in \mathbb{C}^{L_{R} \times L_{T}}$ for $i \in\left\{1,2, \cdots, \frac{N_{R}}{L_{R}}\right\}$ and $j \in\left\{1,2, \cdots, \frac{N_{T}}{L_{T}}\right\}$.

Assume that the number of the available training blocks is $M_{T}$ and the training data for $\boldsymbol{H}_{e s t}^{(i, j)}$ are arranged as

$$
\begin{aligned}
\boldsymbol{Y}_{\mathrm{t} M_{T}}^{(i, j)} & =\left[\boldsymbol{Y}^{(i, j)}(1) \boldsymbol{Y}^{(i, j)}(2) \cdots \boldsymbol{Y}^{(i, j)}\left(M_{T}\right)\right], \\
\boldsymbol{S}_{\mathrm{t} M_{T}}^{(i, j)} & =\left[\boldsymbol{S}^{(i, j)}(1) \boldsymbol{S}^{(i, j)}(2) \cdots \boldsymbol{S}^{(i, j)}\left(M_{T}\right)\right] .
\end{aligned}
$$

Typically, $M_{T}$ is very small. The LSCE of $\boldsymbol{H}_{\text {est }}^{(i, j)}$ based on the training data of (19) and (20) is given by

$$
\widehat{\boldsymbol{H}}_{e s t}^{(i, j)}=\boldsymbol{Y}_{\mathrm{t} M_{T}}^{(i, j)}\left(\boldsymbol{S}_{\mathrm{t} M_{T}}^{(i, j)}\right)^{\mathrm{H}}\left(\boldsymbol{S}_{\mathrm{t} M_{T}}^{(i, j)}\left(\boldsymbol{S}_{\mathrm{t} M_{T}}^{(i, j)}\right)^{\mathrm{H}}\right)^{-1},
$$

and the estimate of $\boldsymbol{H} \in \mathbb{C}^{N_{R} \times N_{T}}$ is expressed as

$$
\widehat{\boldsymbol{H}}=\left[\begin{array}{cccc}
\widehat{\boldsymbol{H}}_{\text {est }}^{(1,1)} & \widehat{\boldsymbol{H}}_{\text {est }}^{(1,2)} & \ldots & \widehat{\boldsymbol{H}}_{\text {est }}^{\left(1, \frac{N_{T}}{L_{T}}\right)} \\
\widehat{\boldsymbol{H}}_{\text {est }}^{(2,1)} & \widehat{\boldsymbol{H}}_{\text {est }}^{(2,2)} & \ldots & \widehat{\boldsymbol{H}}_{\text {est }}^{\left(2, \frac{N_{T}}{L_{T}}\right)} \\
\vdots & \vdots & \ldots & \vdots \\
\widehat{\boldsymbol{H}}_{\text {est }}^{\left(\frac{N_{R}}{L_{R}}, 1\right)} & \widehat{\boldsymbol{H}}_{\text {est }}^{\left(\frac{N_{R}}{L_{R}}, 2\right)} & \ldots & \widehat{\boldsymbol{H}}_{\text {est }}^{\left(\frac{N_{R}}{L_{R}}, \frac{N_{T}}{L_{T}}\right)}
\end{array}\right] .
$$

Then the NBJTRAS is carried out based on this estimated full channel matrix $\widehat{\boldsymbol{H}} \in \mathbb{C}^{N_{R} \times N_{T}}$, which also yields the rough estimate $\widehat{\boldsymbol{H}}_{\text {sub }}$ of the selected subset channel matrix.

\section{B. Tier Two: DDCE}

With a small training blocks $M_{T}$, the accuracy of the estimate $\widehat{\boldsymbol{H}}_{\text {sub }}$ is poor. Note that data detection is more sensitive to the CE error than the NBJTRAS. In the tier two, we use the semi-blind joint $\mathrm{CE}$ and data detection scheme of [24] which applies the DDCE to refine the initial TBCE $\widehat{\boldsymbol{H}}_{\text {sub }}$. Let the observation data at the receiver be

$$
\boldsymbol{Y}_{d}=[\boldsymbol{Y}(1) \boldsymbol{Y}(2) \cdots \boldsymbol{Y}(\tau)],
$$

where $\tau$ is the number of the received data blocks per frame. Given the maximum number of DDCE iterations $I_{\max }$, the tier-two DDCE is summarized as follows.

1) Set the DDCE iteration index as ite $=0$ and the initial $\mathrm{CE}$ as the TBCE of $\widehat{\boldsymbol{H}}_{\text {sub }}: \widehat{\boldsymbol{H}}_{\text {sub }}^{(i t e)}=\widehat{\boldsymbol{H}}_{\text {sub }}$.

2) Perform the ML data detection for $\boldsymbol{Y}_{d}$ based on the $\mathrm{CE} \widehat{\boldsymbol{H}}_{\text {sub }}^{(i t e)}$, and re-modulate the detected data into the symbol sequence given by

$$
\widehat{\boldsymbol{S}}_{d}^{(i t e)}=\left[\widehat{\boldsymbol{S}}^{(i t e)}(1) \widehat{\boldsymbol{S}}^{(i t e)}(2) \cdots \widehat{\boldsymbol{S}}^{(i t e)}(\tau)\right] .
$$

Then the DDCE is updated according to

$$
\widehat{\boldsymbol{H}}_{\text {sub }}^{(i t e+1)}=\boldsymbol{Y}_{d}\left(\widehat{\boldsymbol{S}}_{d}^{(i t e)}\right)^{\mathrm{H}}\left(\widehat{\boldsymbol{S}}_{d}^{(i t e)}\left(\widehat{\boldsymbol{S}}_{d}^{(i t e)}\right)^{\mathrm{H}}\right)^{-1},
$$

3) If ite $=I_{\max }$, stop; else, set ite $=i t e+1$ and go to 2 ). $I_{\max } \leq 4$ is sufficient for this DDCE process to converge.

\section{Simulation Results}

A quasi-static Rayleigh fading $\operatorname{STSK}\left(L_{T}=2, L_{R}=\right.$ $\left.2, T_{n}=2, Q=4, L=4\right)$ was simulated. Various values of $N_{T}$ and $N_{R}$ were used to yield different AS factors $f_{A S}\left(N_{T}, N_{R}\right)$. The transmitted signal power was normalised to unity and thus the SNR was given by $\frac{1}{N_{0}}$. The frame length was set to 1,000 bits, yielding $\tau=250 \operatorname{STSK}(2,2,2,4,4)$ symbol blocks. Two metrics were used to assess the achievable performance, and they were the BER and the mean channel error (MCE) of the CE defined by

$$
J_{\mathrm{MCE}}\left(\widehat{\boldsymbol{H}}_{\text {sub }}\right)=\left\|\boldsymbol{H}_{\text {sub }}-\widehat{\boldsymbol{H}}_{\text {sub }}\right\|^{2} /\left\|\boldsymbol{H}_{\text {sub }}\right\|^{2} .
$$

All the results were averaged over 10,000 channel realizations.

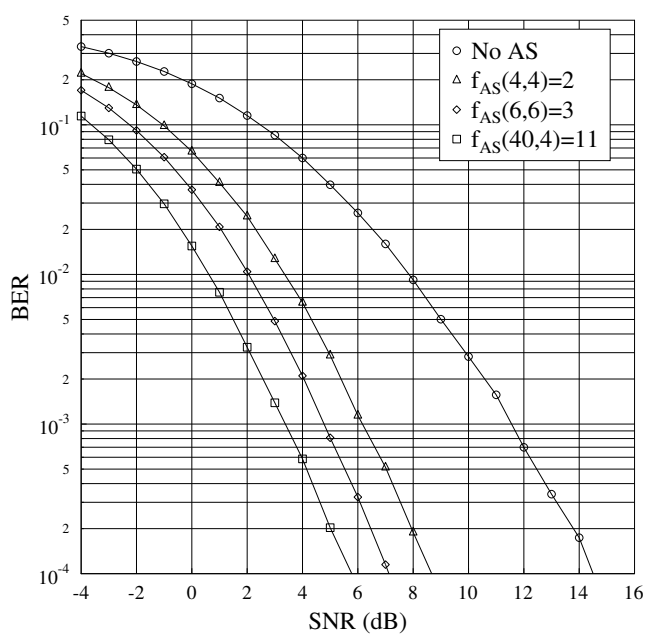

Fig. 2: BER performance of the proposed NBJTRAS aided $\operatorname{STSK}(2,2,2,4,4)$ given three AS factors $f_{A S}\left(N_{T}, N_{R}\right)$, in comparison to performance of the conventional $\operatorname{STSK}(2,2,2,4,4)$ without AS, assuming the perfect CSI at both transmitter and receiver.

1) NBJTRAS Aided STSK with Perfect CSI: The BER of the proposed NBJTRAS aided STSK system is depicted in Fig. 2, in comparison to that of the conventional STSK system without AS. It is seen that given perfect CSI, the NBJTRAS aided STSK system significantly enhances the achievable BER. In particular, at the BER level of $10^{-4}$, the NBJTRAS aided STSK with $f_{A S}(4,4)=2$ achieves a SNR gain of about $6 \mathrm{~dB}$ over the conventional STSK system without AS. When the value of the AS factor increases from 2 to 3, an additional SNR gain of $0.9 \mathrm{~dB}$ is obtained. However, when the AS factor increases from 3 to 11, only an additional SNR gain of $0.8 \mathrm{~dB}$ is achieved. Further increasing the value of the AS factor leads to negligible "diversity" gain attained.

2) Conventional TBCE for NBJTRAS Aided STSK: In order to demonstrate the power of the TTCE scheme, we first tested the conventional TBCE scheme, namely, the NBJTRAS aided $\operatorname{STSK}(2,2,2,4,4)$ employing only the tier-one TBCE scheme. The AS factor $f_{A S}(4,4)=2$ was adopted. The BER achieved by this NBJTRAS aided STSK system with the tier-one TBCE scheme is shown in Fig. 3, where it can be seen that as the number of the STSK training blocks increases, the BER performance is enhanced due to the improved CE accuracy. 


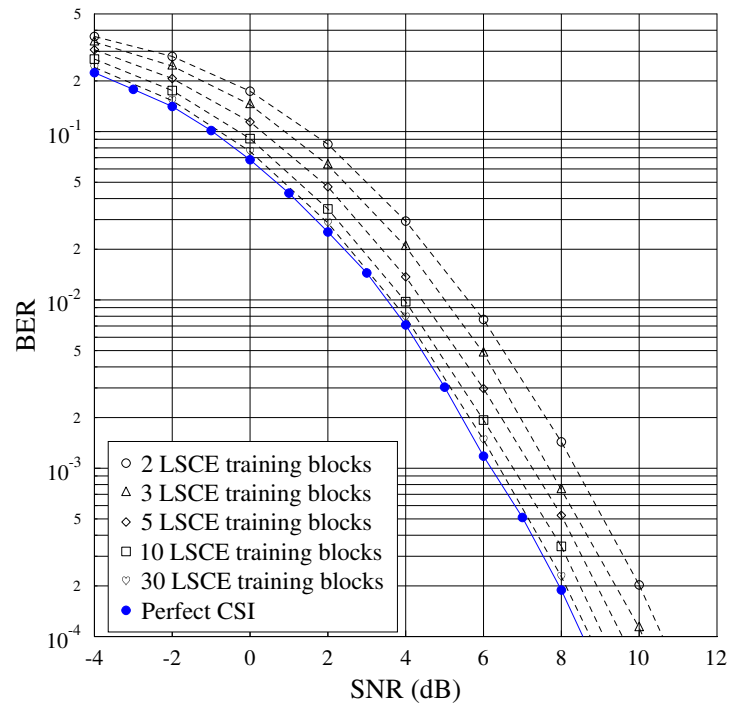

Fig. 3: BER performance of the NBJTRAS aided $\operatorname{STSK}(2,2,2,4,4)$ with $f_{A S}(4,4)=2$, assisted by the conventional TBCE scheme given the number of the STSK training blocks $M_{T}=2,3,5,10$ and 30, in comparison to the perfect CSI case.

When the number of the training blocks increases to $M_{T}=30$, the BER performance gap between the case of perfect CSI and the TBCE based system is smaller than $0.1 \mathrm{~dB}$.

Fig. 4 shows the MCE performance of the NBJTRAS aided STSK system employing the tier-one TBCE scheme, in comparison to the TBCE aided conventional $\operatorname{STSK}(2,2,2,4,4)$ without AS. It can be seen from Fig. 4 that for the cases of $M_{T}=2$ and SNR $<-1 \mathrm{~dB}$ as well as $M_{T}=3$ and SNR $<-3 \mathrm{~dB}$, the MCE of the training based NBJTRAS aided STSK system is slightly worse than that of the non-AS based conventional STSK system. However, when the SNR is larger than $-1 \mathrm{~dB}$, the TBCE based NBJTRAS aided STSK outperforms the TBCE assisted conventional STSK without AS. Moreover, when the training length increases to $M_{T} \geq 5$, the TBCE based NBJTRAS aided STSK system outperforms

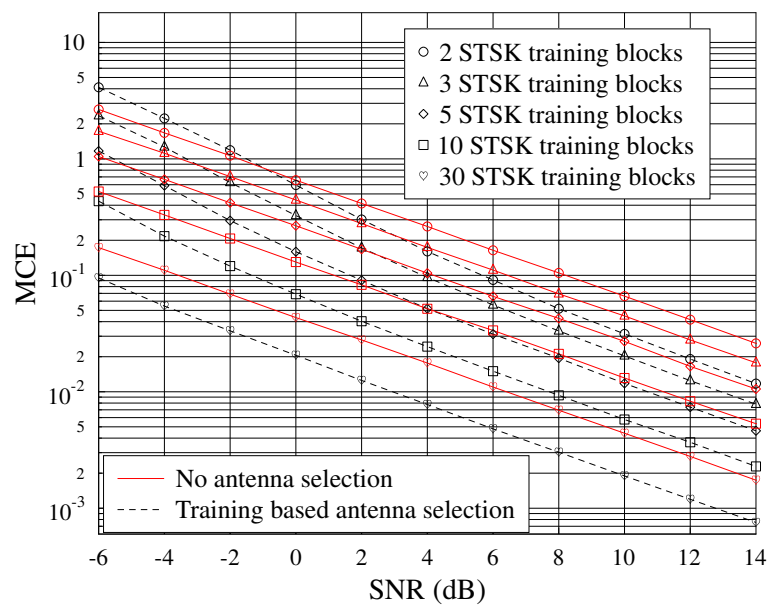

Fig. 4: MCE performance of the NBJTRAS aided $\operatorname{STSK}(2,2,2,4,4)$ with $f_{A S}(4,4)=2$ and employing the conventional TBCE scheme, in comparison to the performance of the TBCE aided conventional $\operatorname{STSK}(2,2,2,4,4)$ without AS. the conventional STSK without AS over the entire SNR range tested. This clearly demonstrates that with the aid of the NBJTRAS scheme, the CE accuracy is improved.

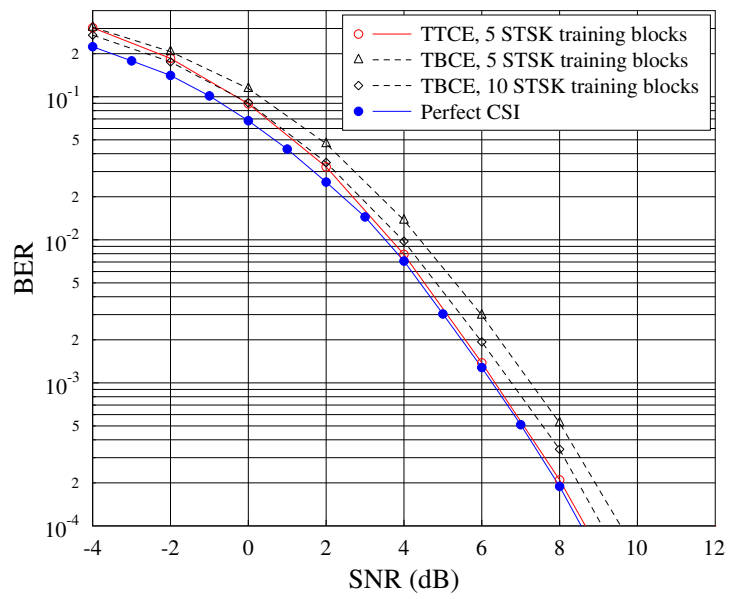

Fig. 5: BER performance of the proposed TTCE based NBJTRAS aided $\operatorname{STSK}(2,2,2,4,4)$ with $M_{T}=5$, in comparison to that of the conventional TBCE assisted NBJTRAS aided $\operatorname{STSK}(2,2,2,4,4)$ with $M_{T}=5$ and 10. $f_{A S}(4,4)=2$ is adopted for the both systems.

3) Proposed TTCE for NBJTRAS Aided STSK: The BER of the proposed TTCE assisted NBJTRAS aided STSK system with $M_{T}=5$ is shown in Fig. 5. It is seen that in the low SNR region of $\mathrm{SNR}<3 \mathrm{~dB}$, the TTCE assisted system fails to converge to the perfect CSI bound. However, for SNR $>3 \mathrm{~dB}$, the BER of the TTCE assisted system is capable of converging to the perfect CSI case. Fig. 5 also shows that the performance of the TBCE assisted NBJTRAS aided STSK system with $M_{T}=10$ is unable to attain the BER of the NBJTRAS aided STSK system associated with perfect CSI.

Fig. 6 illustrates the MCE convergence behaviour of the proposed TTCE scheme for the NBJTRAS aided STSK system with $M_{T}=5$, where it is seen that three iterations are sufficient for the TTCE to converge.

Fig. 7 compares the MCE of the proposed TTCE scheme with $M_{T}=5$ training blocks with those of the conventional TBCE scheme given various numbers of training blocks. As expected, the TBCE assisted NBJTRAS aided STSK system with $M_{T}=5$ training blocks has the same MCE performance

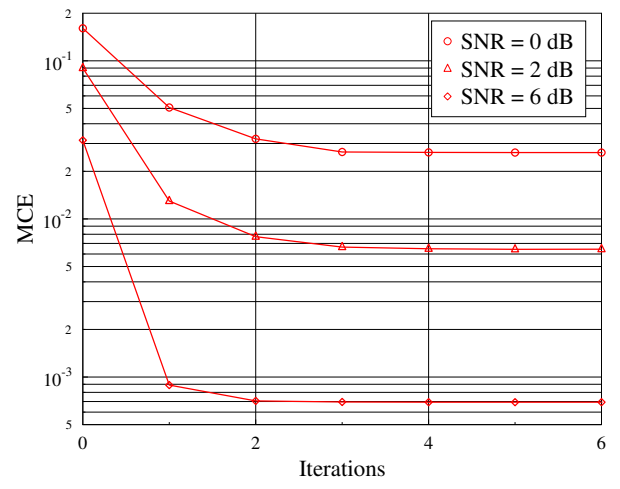

Fig. 6: MCE convergence performance of the proposed TTCE for the NBJTRAS aided $\operatorname{STSK}(2,2,2,4,4)$ with $f_{A S}(4,4)=2$ and $M_{T}=5$ for three SNR values. 


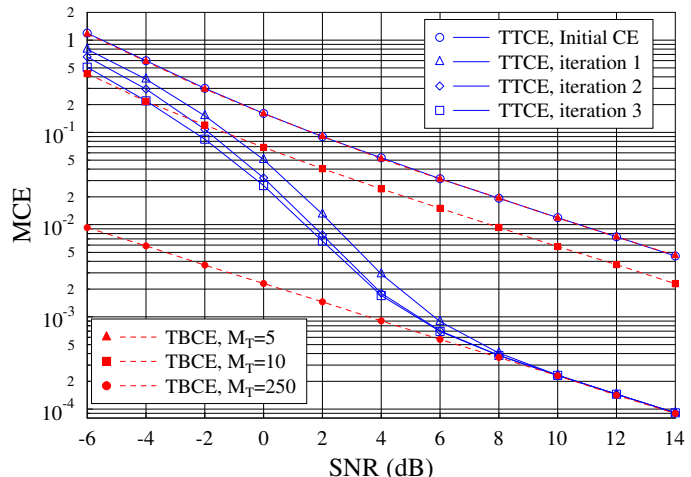

Fig. 7: MCE performance of the proposed TTCE for the NBJTRAS aided $\operatorname{STSK}(2,2,2,4,4)$ with $M_{T}=5$, in comparison to that of the conventional TBCE scheme for the NBJTRAS aided $\operatorname{STSK}(2,2,2,4,4)$ with $M_{T}=5,10$ and 250. $f_{A S}(4,4)=2$ is adopted for the both systems.

as the initial CE of the proposed TTCE assisted NBJTRAS aided STSK system with the same $M_{T}=5$ training blocks. From Fig. 7, it can be seen that the MCE of the TTCE assisted NBJTRAS aided STSK system with only $M=5$ training blocks is capable of converging in three iterations to the MCE of the conventional TBCE assisted NBJTRAS aided STSK system with $M_{T}=250$ training blocks for SNR $>6 \mathrm{~dB}$. For SNR $>6 \mathrm{~dB}$, the BER of the TTCE assisted NBJTRAS aided STSK system is below $10^{-3}$, as can be seen from Fig. 5, and all the $\tau=250$ decisions become reliable. Therefore, the proposed TTCE scheme with $\tau$ blocks per frame is capable of approaching the performance bound of TBCE with $M_{T}=\tau$ training blocks in high SNR region.

\section{CONCLUSIONS}

We have proposed a simple yet efficient NBJTRAS aided STSK system which is capable of significantly outperforming the conventional STSK system without AS, given CSI. Additionally, we have proposed a novel TTCE scheme for assisting the NBJTRAS aided STSK system. The proposed TTCE scheme only requires a very small number of training blocks in the tier one to provide a rough CE for the NBJTRAS to carry out the AS. In the tier two, the selected subset training based $\mathrm{CE}$ is used for initial data detection, and the detected data are re-modulated for further DDCE. Our simulation results have showed that typically 3 iterations are sufficient for this DDCE to converge. Our results have also demonstrated that the proposed TTCE assisted NBJTRAS aided system with a very small number of training blocks is capable of approaching the optimal ML performance bound associated with perfect CSI, provided that the SNR is over certain threshold.

\section{REFERENCES}

[1] S. Sanayei and A. Norsratinia, "Antenna selection in MIMO systems," IEEE Communications Magazine, vol. 42, no. 10, pp. 68-73, Oct. 2004.

[2] R. Rajashekar, K. S. V. Hari, and L. Hanzo, "Antenna selection in spatial modulation systems," IEEE Communications Letters, vol. 17, no. 3, pp. 521-524, March 2013.

[3] W.-H. Chung and C.-Y. Hung, "Multi-antenna selection using space shift keying in MIMO systems," in Proc. VTC2012-Spring (Yokohama, Japan), May 6-9, 2012, pp. 1-5.
[4] S. Kim, M. Shin, and C. Lee, "Transmit antenna selection scheme for iterative receivers in MIMO systems," IEEE Signal Processing Letters, vol. 14, no. 12, pp. 916-919, Dec. 2007.

[5] A. S. Hiwale, A. A. Ghatol, and S. D. Bhad, "Performance analysis of space-time trellis codes with receive antenna selection," in Proc. 4th Int. Conf. Wireless Communication and Sensor Networks (Allahabad, India), Dec. 27-29, 2008, pp. 148-152.

[6] D. Liu and D. K. C. So, "Performance based receive antenna selection for V-BLAST systems," IEEE Trans. Wireless Communications, vol. 8, no. 1, pp. 214-225, Jan. 2009.

[7] Z. Xu, S. Sfar, and R. S. Blum, "Analysis of MIMO systems with receive antenna selection in spatially correlated Rayleigh fading channels," IEEE Trans. Vehicular Technology, vol. 58, no. 1, pp. 251-262, Jan. 2009.

[8] W. A. Al-Hussaibi and F. H. Ali, "Receive antenna selection for uplink multiuser MIMO systems over correlated rayleigh fading channels," in Proc. 14th Int. Symp. Wireless Personal Multimedia Communications (Brest, France), Oct. 3-7, 2011, pp. 1-5.

[9] R. S. Blum, Z. Xu, and S. Sfar, "A near-optimal joint transmit and receive antenna selection algorithm for MIMO systems," in Proc. Radio and Wireless Symposium 2009 (San Diego, USA), Jan. 18-22, 2009, pp. 554-557.

[10] T. Gucluoglu and T. M. Duman, "Space-time coded systems with joint transmit and receive antenna selection," in Proc. ICC-2007 (Glasgow, UK), June 24-28, 2007, pp. 5305-5310.

[11] H. S. Kim and Y. S. Byun, "Near-optimal selection of joint transmitreceive antennas for MIMO channel based on maximizing channel capacity," in Proc. APCC-2006 (Busan, Korea), Aug. 31- Sept. 1, 2006, pp. $1-5$.

[12] M. Naeem and D. C. Lee, "Near-optimal selection of transmit and receive antennas for MIMO systems," in Proc. ISCIT-2009 (Incheon, Korea), Sept. 28-30, 2009, pp. 572-577.

[13] Y. R. Wei and M. Z. Wang, "Capacity-based efficient joint transmit and receive antenna selection schemes in MIMO systems," in Proc. ICTTA2006 (Damascus, Syria), April 24-28, 2006, pp. 2125-2129.

[14] S. Sanayei and A. Nosratinia, "Capacity maximizing algorithms for joint transmit-receive antenna selection," in Proc. 38th Asilomar Conf. Signals, Systems and Computers (California, USA), Nov. 7-10, 2004, pp. 1773-1776.

[15] A. Yilmaz and O. Kucur, "Error performance of joint transmit and receive antenna selection in two hop amplify-and-forward relay system over Nakagami-m fading channels," in Proc. 21th PIMRC (Istanbul, Turkey), Sept. 26-29, 2010, pp. 2198-2203.

[16] T. Gucluoglu and T. M. Duman, "Performance analysis of transmit and receive antenna selection over flat fading channels," IEEE Trans. Wireless Communications, vol. 7, no. 8, pp. 3056-3065, Aug. 2008.

[17] M. Gharavi-Alkhansari and A. B. Gershman, "Fast antenna subset selection in MIMO systems," IEEE Trans. Signal Processing, vol. 52, no. 2, pp. 339-347, Feb. 2004.

[18] Q. Ma and C. Tepedelenlioglu, "Antenna selection for space-time coded systems with imperfect channel estimation," IEEE Trans. Wireless Communications, vol. 6, no. 2, pp. 710-719, Feb. 2007.

[19] A. B. Narasimhamurthy and C. Tepedelenlioglu, "Antenna selection for MIMO-OFDM systems with channel estimation error," IEEE Trans. Wireless Communications, vol. 58, no. 5, pp. 2269-2278, June 2009.

[20] R. Y. Mesleh, H. Haas, S. Sinanovic, C. W. Ahn, and S. Yun, "Spatial modulation," IEEE Trans. Vehicular Technology, vol. 57, no. 4, pp. 2228-2241, July 2008.

[21] J. Jeganathan, A. Ghrayeb, L. Szczecinski, and A. Ceron, "Space shift keying modulation for MIMO channels," IEEE Trans. Wireless Communications, vol. 8, no. 7, pp. 3692-3703, July 2009.

[22] S. Sugiura, S. Chen, and L. Hanzo, "Coherent and differential space-time shift keying: A dispersion matrix approach," IEEE Trans. Communications, vol. 58, no. 10, pp. 3219-3230, Nov. 2010.

[23] P. Zhang, S. Chen, and L. Hanzo, "Reduced-complexity near-capacity joint channel estimation and three-stage turbo detection for coherent space-time shift keying," IEEE Trans. Communications, vol. 61, no. 5, pp. 1902-1912, May 2013.

[24] P. Zhang, I. Dey, S. Sugiura, and S. Chen, "Semi-blind adaptive spacetime shift keying systems based on iterative channel estimation and data detection," in Proc. VTC2011-Spring (Budapest, Hungary), May 15-18, 2011, pp. 1-5.

[25] T. Gucluoglu and E. Panayirci, "Performance of transmit and receive antenna selection in the presence of channel estimation errors," IEEE Communications Letters, vol. 12, no. 5, pp. 371-373, May 2008. 